\title{
ERROR ESTIMATE IN AN ISOPARAMETRIC FINITE ELEMENT EIGENVALUE PROBLEM
}

\author{
M. P. LEBAUD
}

\begin{abstract}
The aim of this paper is to obtain an eigenvalue approximation for elliptic operators defined on a domain $\Omega$ with the help of isoparametric finite elements of degree $k$. We prove that $\lambda-\lambda_{h}=O\left(h^{2 k}\right)$ provided the boundary of $\Omega$ is well approximated, which is the same estimate as the one obtained in the case of conforming finite elements.
\end{abstract}

\section{INTRODUCTION}

We consider a spectral approximation by the isoparametric finite element method for an elliptic operator $L$ defined over a bounded domain $\Omega$ of $\mathbb{R}^{2}$. The goal is to approximate a simple real eigenvalue $\lambda$ of $L$.

J. E. Osborn [10] developed a general spectral approximation theory for compact operators on a Banach space. He proved that the conforming finite element method of degree $k$ made up over a polygonal domain $\Omega$ satisfies the following result:

$$
\left\|u-u_{h}\right\|_{L^{2}(\Omega)}=O\left(h^{k+1}\right) \text { and }\left|\lambda-\lambda_{h}\right|=O\left(h^{2 k}\right) \text {, }
$$

where $(\lambda, u)$ is an eigenpair of an elliptic operator. U. Banerjee and J. E. Osborn [4] took into account the effect of numerical integration and showed that it depends on the degree of precision of the quadrature rules and on the smoothness of the eigenfunctions. To be more precise, they found the same rate of convergence as indicated before if the quadrature rules are of degree $2 k-1$ and $u$ is regular enough. U. Banerjee [3] improved in some way this result: for quadrature rules of degree $2 k-2$, the estimate for the eigenfunction remains true but not for the eigenvalue, where one degree is lost.

For selfadjoint problems, estimate (1.1) has been obtained by several authors; in particular, [5] proved it for Sturm-Liouville problems approximated with piecewise cubic polynomials. It is a one-dimensional paper but it presents a result estimating eigenvalue error in terms of approximability error, which is used for selfadjoint problems in higher dimensions.

If we apply the general results of Osborn [10] to the usual isoparametric finite element approximation over some bounded domains (see $\S 4$ ), we obtain the same rate of convergence as in (1.1) for the eigenfunction $u$ but for the

Received by the editor January 19, 1993 and, in revised form, May 24, 1993.

1991 Mathematics Subject Classification. Primary 65N15, 65N25, 65N30.

Key words and phrases. Elliptic operators, approximation of eigenvalues, isoparametric finite elements.

(C) 1994 American Mathematical Society $0025-5718 / 94 \$ 1.00+\$ .25$ per page 
eigenvalue we only have $\left|\lambda-\lambda_{h}\right|=O\left(h^{k+1}\right)$ because $\partial \Omega$ and $\partial \Omega_{h}$ differ by at most $O\left(h^{k+1}\right)$ (Lemma 3.1). Our purpose in this article is to give a "good" construction of the approximate boundary that will lead to the phenomenon of supraconvergence: $\left|\lambda-\lambda_{h}\right|=O\left(h^{2 k}\right)$. To be more accurate, this estimate can be derived from Theorem 3 in [10] together with (4.4) and the inequality

$$
\ell^{*}\left(\left(T-T_{h}\right) u\right) \leqslant C h^{2 k},
$$

where $\ell^{*}$ is a linear form defined in (2.5). This last estimate involves a careful analysis of the underlying isoparametric approximation and is proved under Hypothesis $(\mathrm{H})$ given in $\S 4$.

In $\S 2$, we briefly describe the exact problem and the approximate one. In $\S 3$, we show how we build up the mesh over the bounded domain $\Omega$ of interest and how we devise the external layer of the elements to obtain a good approximation of the boundary $\partial \Omega$. The main result is given in $\S 4$, where we also recall some previous results we need next. This result is proved in two steps: first we write $\lambda-\lambda_{h}$ as an integral defined over $\partial \Omega(\S 5)$; then the estimate of this integral (§6) leads to the result. In the last section, some examples of triangulations satisfying the requirements of the theorem are given in the cases $k=2$ and $k=3$.

\section{SETTING FOR PROBLEM}

Let $\Omega$ be a bounded domain of $\mathbb{R}^{2}$ with a $C^{\infty}$-boundary $\partial \Omega$. We define an operator $L$ on $C^{2}(\bar{\Omega})$ by

$$
L u=-\sum_{i, j=1}^{2} \frac{\partial}{\partial x_{j}}\left(a_{i j} \frac{\partial u}{\partial x_{i}}\right),
$$

where $a_{i j}$ belong to $C^{\infty}\left(\mathbb{R}^{2}, \mathbb{R}\right)$. We assume that $L$ is uniformly strongly elliptic, i.e., there is a constant $a_{0}>0$ such that

$$
\forall \xi \in \mathbb{R}^{2}, \forall x \in \mathbb{R}^{2} \quad \sum_{i, j=1}^{2} a_{i j}(x) \xi_{i} \xi_{j} \geqslant a_{0} \sum_{i=1}^{2} \xi_{i}^{2} .
$$

We associate with $L$ the following bilinear form defined on $H^{1}(\Omega) \times H^{1}(\Omega)$ :

$$
a_{\Omega}(u, v)=\sum_{i, j=1}^{2} \int_{\Omega} a_{i j}(x) \frac{\partial u}{\partial x_{i}} \frac{\partial v}{\partial x_{j}} d x .
$$

It is coercive on $H_{0}^{1}(\Omega) \times H_{0}^{1}(\Omega)$; furthermore, the boundedness of $a_{i j}$ on $\bar{\Omega}$ implies that $a_{\Omega}$ is continuous on $H^{1}(\Omega)$. According to the Lax-Milgram theorem, the problem

$$
\left\{\begin{array}{l}
\text { for } f \in L^{2}(\Omega), \quad \text { find } u \in H_{0}^{1}(\Omega) \text { such that } \\
a_{\Omega}(u, \varphi)=\int_{\Omega} f(x) \varphi(x) d x \text { for all } \varphi \in H_{0}^{1}(\Omega)
\end{array}\right.
$$

has one and only one solution $u=T f$. The operator $T$ is an operator according to the Rellich theorem. We denote by $\mu$ a nonzero, real and simple eigenvalue of $T$ and by $u$ an associated eigenfunction, normalized with respect to the $L^{2}(\Omega)$ norm. We may then choose an eigenfunction $u^{*}$ of $T^{*}$ 


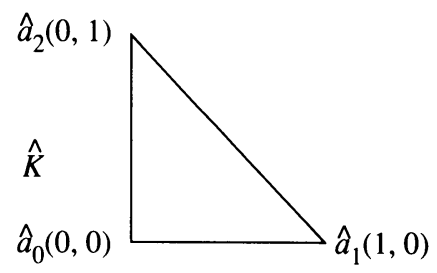

FIGURE 2.1

associated with $\mu$, where $T^{*}$ is the adjoint of $T$ with respect to the $L^{2}(\Omega)$ inner product, in such a way that

$$
\int_{\Omega} u^{*} u d x=1
$$

We consider the following problem:

$$
\left(P_{1}\right) \quad\left\{\begin{aligned}
u-\lambda T u & =0, \\
\ell^{*}(u) & =1,
\end{aligned}\right.
$$

where $\lambda=1 / \mu$ and $\ell^{*}$ is the linear form defined on $L^{2}\left(\mathbb{R}^{2}\right)$ by

$$
\ell^{*}(v) \stackrel{\text { def }}{=} \int_{\Omega} u^{*} v d x .
$$

We assume the space $W^{m, p}(\Omega)$ normed with

$$
\|u\|_{m, p, \Omega}=\left(\sum_{|\alpha| \leqslant m}\left\|\partial^{\alpha} u\right\|_{p}^{p}\right)^{\frac{1}{p}},
$$

where $\|\cdot\|_{p}$ is the usual norm of $L^{p}(\Omega)$. We use also the seminorm

$$
|u|_{m, p, \Omega}=\left(\sum_{|\alpha|=m}\left\|\partial^{\alpha} u\right\|_{p}^{p}\right)^{\frac{1}{p}}
$$

and make the usual changes if $p=\infty$.

We consider the approximation of $\left(P_{1}\right)$ by the isoparametric finite element method of Lagrangian type and start by reviewing the construction of a triangulation associated with this method $([6,7,8])$. Let $k$ be a nonnegative integer and $(\widehat{K}, \widehat{P}, \widehat{\Sigma})$ the finite element of reference defined as follows:

$-\widehat{K}=\left\{\widehat{x}=\left(\widehat{x}_{1}, \widehat{x}_{2}\right) ; \widehat{x}_{1} \geqslant 0 ; \widehat{x}_{2} \geqslant 0 ; \widehat{x}_{1}+\widehat{x}_{2} \leqslant 1\right\}$ is a triangle whose vertices are denoted by $\widehat{a}_{0}, \widehat{a}_{1}, \widehat{a}_{2}$ (see Figure 2.1 ).

$-\widehat{P}=P_{k}$, where $P_{k}$ is the space of all polynomials of degree not exceeding $k$ defined on $\widehat{K}$.

$-\widehat{\Sigma}=\left\{\widehat{x}=\left(\hat{x}_{1}, \hat{x}_{2}\right) ; \hat{x}_{1}=i / k ; \hat{x}_{2}=j / k ; i+j \leqslant k ; i, j \in \mathbb{N}\right\}$, the set of all Lagrangian interpolation nodes.

We consider an open set $\Omega_{h}$ approximating $\Omega$ and a triangulation $\mathscr{K}_{h}$ of curved finite elements: an element $K$ of $\mathscr{K}_{h}$ is given by $K=F_{K}(\widehat{K})$, where $F_{K}$ is an invertible mapping each component of which belongs to $P_{k}$. The map $F_{K}$ is indeed determined by the data of the images $a_{i, K}$ of the nodes $\hat{a}_{i}$ belonging to $\widehat{\Sigma}$. We assume that, if an edge $\Gamma$ of $K$ is on $\partial \Omega_{h}$, its vertices 
$k=2$

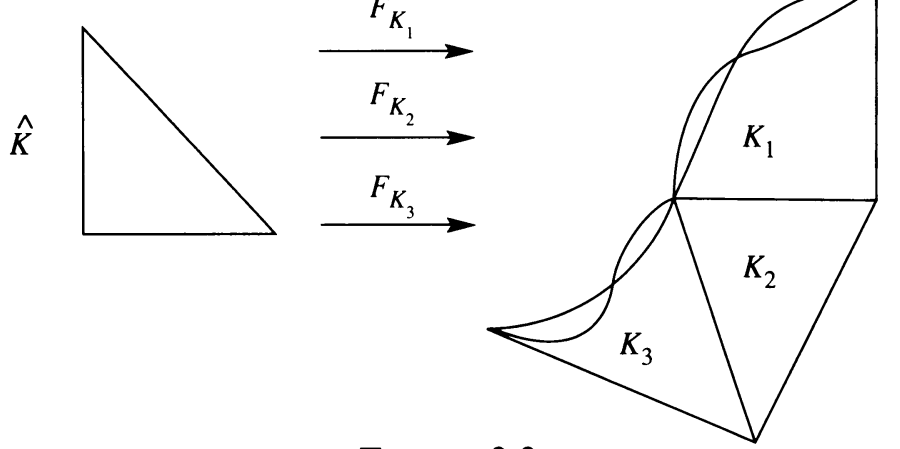

FIGURE 2.2

are on $\partial \Omega$ too and that the edges which do not belong to $\partial \Omega_{h}$ are straight. These hypotheses are illustrated by Figure 2.2 .

We denote by $h_{K}$ the diameter of $K$ and assume that all $h_{K}$ are bounded by $h$.

We define the space of functions $V_{h}$ by

$$
V_{h}=\left\{v \in C^{0}\left(\mathbb{R}^{2}\right) ; v(x)=0 \text { if } x \notin \Omega_{h} ; v_{/ K} \in P_{K} \forall K \in \mathscr{K}_{h}\right\},
$$

where $P_{K}=\left\{p: K \rightarrow \mathbb{R} ; p \circ F_{K} \in P_{k}\right\}$. It is easy to check that

$$
V_{h} \subset H_{0}^{1}\left(\Omega_{h}\right) \text {. }
$$

We also assume that this triangulation is $k$-regular (Ciarlet and Raviart [7]). We now approximate our problem. We first define an elliptic bilinear form on $V_{h} \times V_{h}$ by

$$
a_{h}\left(v_{h}, w_{h}\right)=\sum_{i, j=1}^{2} \int_{\Omega_{h}} a_{i j} \frac{\partial v_{h}}{\partial x_{i}} \frac{\partial w_{h}}{\partial x_{j}} d x
$$

We also define two operators $T_{h}$ and $T_{h}^{*}$ from $L^{2}\left(\mathbb{R}^{2}\right)$ to $V_{h}$ by

$$
\forall f \in L^{2}\left(\mathbb{R}^{2}\right), \forall v_{h} \in V_{h}\left\{\begin{array}{l}
a_{h}\left(T_{h} f, v_{h}\right)=\int_{\mathbb{R}^{2}} f v_{h} d x, \\
a_{h}\left(v_{h}, T_{h}^{*} f\right)=\int_{\mathbb{R}^{2}} f v_{h} d x,
\end{array}\right.
$$

and $u_{h}$ and $\lambda_{h}$ are solutions of

$$
\left(P_{2}\right) \quad u_{h}-\lambda_{h} T_{h} u_{h}=0 .
$$

We furthermore assume that $u_{h}$ is the orthogonal projection of $u$ on the eigenspace of $T_{h}$ associated with $\mu_{h}=1 / \lambda_{h}$. We then derive an estimate for $\lambda-\lambda_{h}$.

Remark. Most of the time, $\Omega$ and $\Omega_{h}$ are different. We sometimes need to extend functions defined on $\Omega$ or $\Omega_{h}$ to $\mathbb{R}^{2}$ in a continuous way and use the 
same notation for a function and its extension. Unless explicitly mentioned, an $H_{0}^{1}(\Omega)$-function is extended by zero outside of $\Omega$.

\section{Curved triangles}

We shall obtain the stated estimate, $\lambda-\lambda_{h}=O\left(h^{2 k}\right)$, by means of "good approximation" of the boundary $\partial \Omega$. This needs explanation, which we provide in this section.

We assume that $\partial \Omega$ is parametrized by its arclength $\sigma \rightarrow x(\sigma)$ and denote by $\vec{n}(\sigma)$ the unitary normal vector, exterior to $\partial \Omega$ at the point $x(\sigma)$ and by $L$ the length of $\partial \Omega$.

Consider the mapping defined as follows:

$$
\chi:(\sigma, \xi) \rightarrow \chi(\sigma, \xi)=x(\sigma)+\xi \vec{n}(\sigma) .
$$

If $a>0$ is small enough, $\chi$ is a $C^{\infty}$-diffeomorphism from [0,L] $\times[-a, a]$ onto a neighborhood $\mathscr{V}$ of $\partial \Omega$ in $\mathbb{R}^{2}$. From now on, we assume that $h$ is small enough so that

$$
\partial \Omega_{h} \subset \mathscr{V} .
$$

Remark. If $M=x(\sigma)+\xi \vec{n}(\sigma) \in \mathscr{V}$, then $x(\sigma)$ is the orthogonal projection of $M$ on $\partial \Omega$ and $|\xi|=d(M, \partial \Omega)$, where $d(M, \partial \Omega)$ is the distance of $M$ to $\partial \Omega$.

Now let us consider $K$ a triangle of $\mathscr{K}_{h}$, with a curved edge $\Gamma_{h}$ in $\partial \Omega_{h}$ and let $a_{0}=x\left(\sigma_{i}\right)$ and $a_{1}=x\left(\sigma_{i+1}\right)$ be the vertices of $\Gamma_{h}$. We call $\Gamma$ the part of $\partial \Omega$ lying between those two points, and we denote by $l_{i}=\sigma_{i+1}-\sigma_{i}$ its length. We remark that

$$
l_{i}=O(h) .
$$

We assume that $a_{0}=F_{K}\left(\widehat{a}_{0}\right)$ and $a_{1}=F_{K}\left(\widehat{a}_{1}\right)$, where $F_{K}$ is the mapping of $\left(P_{k}\right)^{2}$ that defines $K$; thus, $\Gamma_{h}$ is the image of the segment $\left[\hat{a}_{0}, \widehat{a}_{1}\right]$ under $F_{K}$, and letting

$$
x_{h}(\sigma)=F_{K}\left(\frac{\sigma-\sigma_{i}}{l_{i}}, 0\right),
$$

we obtain a parametrized equation of $\Gamma_{h}$. Furthermore, $x_{h}$ is a polynomial of degree $k$ with respect of $\sigma$ on $\left[\sigma_{i-1}, \sigma_{i}\right]$.

We assumed that for every $i$

$$
x_{h}\left(\sigma_{i}\right)=x\left(\sigma_{i}\right) .
$$

We furthermore assume that there is a constant $C>0$ such that, for all $i$, we have

$$
\left|x_{h}\left(\sigma_{i}+j \frac{l_{i}}{k}\right)-x\left(\sigma_{i}+j \frac{l_{i}}{k}\right)\right| \leqslant C l_{i}^{k+1} \text { for } j=1, \ldots, k-1 .
$$

Lemma 3.1. Assume that (3.2), (3.5), and (3.6) hold. Then there is a constant $C>0$ such that, for all $i$, we have

$$
\left\|x_{h}-x\right\|_{m, \infty,\left[\sigma_{i}, \sigma_{i+1}\right]} \leqslant C h^{k+1-m} \text { for } m=0, \ldots, k+1 .
$$


Proof. Let $\sigma \rightarrow g_{h} x(\sigma)$ be the Lagrangian interpolation polynomial at the points $\sigma_{i}+j l_{i} / k$ for $j=0, \ldots, k$ of the function $\sigma \rightarrow x(\sigma)$. Thus, we have

$$
\left\{\begin{array}{l}
g_{h} x\left(\sigma_{i}+j l_{i} / k\right)=x\left(\sigma_{i}+j l_{i} / k\right) \text { for } j=0, \ldots, k, \\
g_{h} x \in\left(P_{k}\right)^{2}
\end{array}\right.
$$

It is well known that

$$
\left\|g_{h} x-x\right\|_{m, \infty,\left[\sigma_{i}, \sigma_{i+1}\right]} \leqslant C h^{k+1-m} \text { for } m=0, \ldots, k+1,
$$

with $C$ independent of $i$ and of $h$. We define the Lagrange polynomial basis as follows:

$$
\ell_{j}(\sigma)=\prod_{p \neq j}\left(\frac{\sigma-\sigma_{i}-p l_{i} / k}{(j-p) l_{i} / k}\right) \quad \text { for } j=1, \ldots, k-1 .
$$

Then we can write

$$
g_{h} x(\sigma)-x_{h}(\sigma)=\sum_{m=1}^{k-1}\left(x\left(\sigma_{i}+m l_{i} / k\right)-x_{h}\left(\sigma_{i}+m l_{i} / k\right)\right) \ell_{m}(\sigma) .
$$

The result is thus a consequence of (3.7) and (3.8) and of the well-known estimate

$$
\left|\frac{d^{m}}{d \sigma^{m}} \ell_{j}(\sigma)\right| \leqslant \frac{C}{l_{i}^{m}} \text { for } m=0, \ldots, k+1 .
$$

Remark. We deduce, from this lemma, that the function $x_{h}$ and all its derivatives are bounded on $[0, L]$ independently of $h$.

According to (3.2), we observed that for $\sigma \in[0, L]$ there is a unique $\xi \in(-a, a)$ such that $x(\sigma)+\xi \vec{n}(\sigma) \in \partial \Omega_{h}$. Let $d_{h}(\sigma)$ be this value of $\xi$; and we obtain a new parametrized equation of $\partial \Omega_{h}$ :

$$
\sigma \rightarrow \tilde{x}_{h}(\sigma)=x(\sigma)+d_{h}(\sigma) \vec{n}(\sigma) .
$$

Lemma 3.1 then implies

Corollary 3.1. The mapping $d_{h}$ is $C^{\infty}$ on $\left[\sigma_{i}, \sigma_{i+1}\right]$ for every $i$, and we have

$$
d_{h}(\sigma)=O\left(h^{k+1}\right) \text {. }
$$

Proof. Since $d_{h}(\sigma)=\left(\tilde{x}_{h}(\sigma)-x(\sigma), \vec{n}(\sigma)\right)$, the regularity of $\tilde{x}_{h}$ gives the regularity of $d_{h}$.

Put $\widehat{K}_{\frac{1}{2}}=\{(x, y) \in \widehat{K} ; y \geqslant 1 / 2\}$. There is a constant $h_{0}$ such that, for $h \leqslant h_{0}$, we have $\widehat{K}_{\frac{1}{2}} \subset \widehat{K \cap \Omega}$. From now on, we assume that $h \leqslant h_{0}$.

Lemma 3.2. There is a constant $C_{1}>0$ such that, for all $h \leqslant h_{0}$ and for all $\widehat{v} \in P_{k}$, we have, for $i=0,1$ :

$$
|\widehat{v}|_{i, \infty, \hat{K}} \leqslant C_{1}|\widehat{v}|_{i, 2, \widehat{K \cap \Omega}} .
$$

Proof. $P_{k}$ is a space of finite dimension; thus, by the equivalence of norms, we obtain

We conclude by using $\widehat{K}_{\frac{1}{2}} \subset \widehat{K \cap} \Omega$.

$$
|\widehat{v}|_{i, \infty, \hat{K}} \leqslant C_{1}|\widehat{v}|_{i, 2, \hat{K}_{\frac{1}{2}}}
$$


Lemma 3.3. There is a constant $C>0$ such that, for all $h>0$ small enough and for all $v \in V_{h}$, we have

$$
\|v\|_{1,2, \Delta_{e}} \leqslant C h^{\frac{k}{2}}\|v\|_{1,2, \Omega \cap \Omega_{h}}
$$

where $\Delta_{e}=\Omega_{h} \backslash\left(\Omega \cap \Omega_{h}\right)$.

Proof. Let $\widehat{K \cap \Delta_{e}}=F_{K}^{-1}\left(K \cap \Delta_{e}\right)$ and $J_{K}(\widehat{x})$ be the Jacobian of the mapping $F_{K}$ at the point $\widehat{x}$ of $\widehat{K}$. According to the $k$-regularity of the triangulation ([]]), there is a nonnegative constant $C_{0}$ such that

$$
0<\frac{1}{C_{0}} \leqslant \frac{J_{K}(\widehat{x})}{J_{K}(\widehat{y})} \leqslant C_{0} \text { for all } \widehat{x}, \widehat{y} \in \widehat{K} .
$$

We deduce

$$
\operatorname{area}\left(\widehat{K \cap \Delta_{e}}\right) \leqslant \frac{\operatorname{area}\left(K \cap \Delta_{e}\right)}{\operatorname{area}(K)} \leqslant C h^{k}
$$

since

$$
\left\{\begin{aligned}
\operatorname{area}\left(K \cap \Delta_{e}\right) & \leqslant C h^{k+2}, \\
\operatorname{area}(K) & \geqslant C h^{2}, \\
\operatorname{area}(\widehat{K}) & =1 / 2 .
\end{aligned}\right.
$$

We then consider a function $v$ of $P_{K}$; let $\widehat{v}=v \circ F_{K}$ so that $\widehat{v} \in P_{k}$ thanks to the definition of $P_{K}$. Thus, we can write the following inequalities:

$$
\begin{aligned}
|v|_{0,2, K \cap \Delta_{e}} & \leqslant\left(\max _{\hat{x} \in \hat{K}} J_{K}(\widehat{x})\right)^{\frac{1}{2}}|\widehat{v}|_{0,2, \widehat{K \cap \Delta_{e}}} \\
& \leqslant\left(\max _{\hat{x} \in \hat{K}} J_{K}(\widehat{x})\right)^{\frac{1}{2}}\left(\operatorname{surface}\left(\widehat{K \cap \Delta_{e}}\right)\right)^{\frac{1}{2}}|\widehat{v}|_{0, \infty, \hat{K}} \\
& \leqslant C h^{\frac{k}{2}}\left(\max _{\hat{x} \in \hat{K}} J_{K}(\widehat{x})\right)^{\frac{1}{2}}|\widehat{v}|_{0, \infty, \hat{K}} \text {, according to }(3.11) \\
& \leqslant C h^{\frac{k}{2}}\left(\max _{\hat{x} \in \hat{K}} J_{K}(\widehat{x})\right)^{\frac{1}{2}}|\widehat{v}|_{0,2, \widehat{K \cap \Omega}}, \text { according to Lemma } 3.2 \\
& \leqslant C h^{\frac{k}{2}}\left(\frac{\max _{\hat{x} \in \hat{K}} J_{K}(\widehat{x})}{\min _{\hat{x} \in \hat{K}} J_{K}(\widehat{x})}\right)^{\frac{1}{2}}|v|_{0,2, K \cap \Omega} .
\end{aligned}
$$

The inequality (3.10) then implies

$$
|v|_{0,2, K \cap \Delta_{e}} \leqslant C_{1} h^{\frac{k}{2}}|v|_{0,2, K \cap \Omega}
$$

The $k$-regularity of the triangulation implies also that there is a constant $C$ such that, for all $K \in \mathscr{K}_{h}$, we have

$$
\left\{\begin{array}{c}
\left\|D F_{K}\right\|_{0, \infty, \hat{K}} \leqslant C h \\
\left\|D F_{K}^{-1}\right\|_{0, \infty, K} \leqslant \frac{C}{h}
\end{array}\right.
$$


Furthermore,

$$
\begin{aligned}
& |v|_{1,2, K \cap \Delta_{e}} \leqslant\left(\max _{\hat{x} \in \hat{K}} J_{K}(\widehat{x})\right)^{\frac{1}{2}}\left\|D F_{K}^{-1}\right\|_{0, \infty, K}^{\frac{1}{2}}|\widehat{v}|_{1,2, \widehat{K \cap \Delta_{e}}} \\
& \quad \leqslant C h^{\frac{k}{2}}\left(\max _{\hat{x} \in \hat{K}} J_{K}(\widehat{x})\right)^{\frac{1}{2}}\left\|D F_{K}^{-1}\right\|_{0, \infty, K}^{\frac{1}{2}}|\widehat{v}|_{1, \infty, \hat{K}} \text { according to }(3.11) \\
& \quad \leqslant C h^{\frac{k}{2}}\left(\frac{\max _{\hat{x} \in \hat{K}} J_{K}(\widehat{x})}{\min _{\hat{x} \in \hat{K}} J_{K}(\widehat{x})}\right)^{\frac{1}{2}}\left\|D F_{K}^{-1}\right\|_{0, \infty, K}^{\frac{1}{2}}\left\|D F_{K}\right\|_{0, \infty, \hat{K}}^{\frac{1}{2}}|v|_{1,2, K \cap \Omega},
\end{aligned}
$$

according to Lemma 3.2. The inequalities (3.13) then give

$$
|v|_{1,2, K \cap \Delta_{e}} \leqslant C_{2} h^{\frac{k}{2}}|v|_{1,2, K \cap \Omega} .
$$

Adding up the inequalities (3.12) and (3.14) over all the triangles that are involved, we obtain Lemma 3.3.

Remark. The inequality (3.14) is optimal, but (3.12) could be improved.

\section{The MaIN Result}

We use the notations defined in $\S 3$. Recall that we assume

(1) For all $i$

$$
\left(H_{1}\right) \quad x\left(\sigma_{i}\right)=x_{h}\left(\sigma_{i}\right) .
$$

(2) There is a constant $C>0$ such that for all $j \in\{1, \ldots, k-1\}$ and for all $i$

$$
\left|x_{h}\left(\sigma_{i}+j \frac{l_{i}}{k}\right)-x\left(\sigma_{i}+j \frac{l_{i}}{k}\right)\right| \leqslant C l_{i}^{k+1} .
$$

We denote by $\theta_{0}=0, \ldots, \theta_{k}=1$ the $k+1$ Gauss-Lobatto points of the inierval $[0,1]$ and define

$$
\sigma_{i, j}=\sigma_{i}+\theta_{j} l_{i}, \quad \text { for } j=0, \ldots, k .
$$

Theorem. If $\left(H_{1}\right)$ and $\left(H_{2}\right)$ hold, and if the triangulation $\mathscr{K}_{h}$ is $k$-regular, then there is a constant $M$ independent of $h$ such that

$$
\left|\lambda-\lambda_{h}\right| \leqslant M\left(h^{2 k}+\max _{i, j}\left|x\left(\sigma_{i, j}\right)-x_{h}\left(\sigma_{i, j}\right)\right|\right) .
$$

Remark. If we assume that

$$
\text { ( } H) \quad \max _{i, j}\left|x\left(\sigma_{i, j}\right)-x_{h}\left(\sigma_{i, j}\right)\right|=O\left(h^{2 k}\right),
$$

we obtain the supraconvergence phenomenon

$$
\lambda-\lambda_{h}=O\left(h^{2 k}\right) \text {. }
$$

In order to prove the theorem, we establish the two following propositions.

Proposition 1. There is a constant $C_{1}$ such that

$$
\left|\lambda-\lambda_{h}-\int_{\partial \Omega} g(\sigma) d_{h}(\sigma) d \sigma\right| \leqslant C_{1} h^{2 k},
$$

where $g$ is a regular function of $\sigma$.

We then estimate the integral with 
Proposition 2. Let $\varphi \in W^{k-1,1}(\partial \Omega)$; then there is a constant $C_{2}>0$ such that

$$
\begin{aligned}
\left|\int_{\partial \Omega} \varphi(\sigma) d_{h}(\sigma) d \sigma\right| \leqslant & C_{2} h^{2 k}\left(|\varphi|_{k-1,1, \partial \Omega}+L\|\varphi\|_{k-2, \infty, \partial \Omega}\right) \\
& +C_{2} L\|\varphi\|_{k-2, \infty, \partial \Omega} \max _{i, j}\left|\left(x-x_{h}\right)\left(\sigma_{i, j}\right)\right| .
\end{aligned}
$$

Remark. The first proposition is valid in any dimension of space, but this is not the case for the second one, where the dimension two plays an important role.

These propositions clearly imply the theorem. We shall prove them in the two following sections. For later purposes, we first recall some results.

If the triangulation is $k$-regular, we have

$$
\begin{aligned}
& \text { For all } u \in H^{k+1}\left(\mathbb{R}^{2}\right), \\
& \quad\left\|\left(T-T_{h}\right) u\right\|_{m, 2, \Omega \cap \Omega_{h}} \leqslant C h^{k+1-m}\|T u\|_{k+1,2, \Omega} \text { for } m=0,1 .
\end{aligned}
$$

One can find a proof of this statement in the articles by Zlámal $[12,13]$ in the case of Dirichlet-type problems. It has been improved by Zenisek [11] for various types of nonhomogeneous boundary value problems.

We remark that the definitions of $T$ and $T_{h}$ imply that

$$
\begin{aligned}
\left\|\left(T-T_{h}\right) u\right\|_{0,2, \mathbb{R}^{2}} \leqslant & \left\|\left(T-T_{h}\right) u\right\|_{0,2, \Omega \cap \Omega_{h}}+\left\|\left(T-T_{h}\right) u\right\|_{0,2, \mathbb{R}^{2} \backslash\left(\Omega \cap \Omega_{h}\right)} \\
\leqslant & \left\|\left(T-T_{h}\right) u\right\|_{0,2, \Omega \cap \Omega_{h}}+\left\|T_{h} u\right\|_{0,2, \Omega_{h} \backslash\left(\Omega \cap \Omega_{h}\right)} \\
& +\|T u\|_{0,2, \Omega \backslash\left(\Omega \cap \Omega_{h}\right)}
\end{aligned}
$$

since $T_{h} u=0$ on $\mathbb{R}^{2} \backslash \Omega_{h}$ and $T u=0$ on $\mathbb{R}^{2} \backslash \Omega$. Then, by the Poincaré inequality,

for $u \in H^{k+1}\left(\mathbb{R}^{2}\right)$, such that $T u \in H_{0}^{1}(\Omega)$, we have

$$
\begin{aligned}
\|T u\|_{0,2, \Omega \backslash\left(\Omega \cap \Omega_{h}\right)} & \leqslant C h^{k+1}\|\nabla T u\|_{0,2, \Omega \backslash\left(\Omega \cap \Omega_{h}\right)} \text { according to Lemma } 3.1 \\
& \leqslant C h^{k+1}\|\nabla T u\|_{1,2, \Omega} ;
\end{aligned}
$$

for $u \in H^{k+1}\left(\mathbb{R}^{2}\right)$, such that $T_{h} u \in H_{0}^{1}\left(\Omega_{h}\right)$, we have

$$
\begin{aligned}
\left\|T_{h} u\right\|_{0,2, \Omega_{h} \backslash\left(\Omega \cap \Omega_{h}\right)} & \leqslant C h^{k+1}\left\|\nabla T_{h} u\right\|_{0,2, \Omega_{h} \backslash\left(\Omega \cap \Omega_{h}\right)} \\
& \leqslant C h^{k+1}\left\|T_{h} u\right\|_{1,2, \Omega \backslash\left(\Omega \cap \Omega_{h}\right)} \\
& \leqslant C h^{\frac{3}{2} k+1}\left\|T_{h} u\right\|_{1,2, \Omega \cap \Omega_{h}} \text { according to Lemma } 3.3 \\
& \leqslant C h^{\frac{3}{2} k+1} \text { according to (4.2). }
\end{aligned}
$$

The previous inequalities and (4.3) lead to

$$
\left\|\left(T-T_{h}\right) u\right\|_{0,2, \mathbb{R}^{2}}=O\left(h^{k+1}\right) .
$$

We then use two results from the general theory of the spectral approximation for compact operators by Osborn [10].

Let $T$ be a compact operator of $L^{2}(\Omega)$ into $H_{0}^{1}(\Omega)$. We define a compact operator $\widetilde{T}$ from $L^{2}\left(\mathbb{R}^{2}\right)$ into $L^{2}\left(\mathbb{R}^{2}\right)$ as follows:

Let $u \in L^{2}\left(\mathbb{R}^{2}\right)$; then

$$
\left\{\begin{array}{lll}
\widetilde{T} u=T(u / \Omega) & \text { on } & \Omega, \\
\widetilde{T} u=0 & \text { on } & \mathbb{R}^{2} \backslash \Omega .
\end{array}\right.
$$


The operator $T_{h}$ is from $L^{2}\left(\mathbb{R}^{2}\right)$ into $V_{h}$, thus into $L^{2}\left(\mathbb{R}^{2}\right)$. We denote by $E$ (respectively $E_{h}$ ) the projection of $L^{2}\left(\mathbb{R}^{2}\right)$ onto the space of generalized eigenvectors of $T$ (respectively $T_{h}$ ) corresponding to $\mu$ (respectively $\mu_{h}=$ $\left.1 / \lambda_{h}\right)$. These spaces are spanned respectively by $u$ and $u_{h}$ defined by $\left(P_{1}\right)$ and $\left(P_{2}\right)$. We notice that $u_{h}=E_{h} u$. We let $R(E)$ be the range of the mapping $E$. Given two closed subspaces $M$ and $N$ of $L^{2}\left(\mathbb{R}^{2}\right)$, we set

$$
\begin{aligned}
& \delta(M, N)=\sup \left\{\left\{\inf \left\{\|f-g\|_{0,2, \mathbb{R}^{2}} ; g \in N\right\}\right\} f \in M ;\|f\|_{0,2, \mathbf{R}^{2}=1}\right\}, \\
& \widehat{\delta}(M, N)=\max (\delta(M, N), \delta(N, M)) .
\end{aligned}
$$

Osborn proves in [10] that

There are two constants $C_{1}>0$ and $C_{2}>0$ such that

$$
\left\{\begin{aligned}
\hat{\delta}\left(R(E), R\left(E_{h}\right)\right) & \leqslant C_{1}\left\|\left(T-T_{h}\right)_{/ R(E)}\right\|, \\
\left|\mu-\mu_{h}\right| & \leqslant C_{2}\left\|\left(T-T_{h}\right)_{/ R(E)}\right\| .
\end{aligned}\right.
$$

Moreover,

$$
\begin{aligned}
&\left\|\left(T-T_{h}\right)_{/ R(E)}\right\|=\sup \left\{\left|\left(\left(T-T_{h}\right) f, \varphi\right)\right| ; f \in R(E), \varphi \in L^{2}\left(\mathbb{R}^{2}\right) ;\right. \\
&\left.\|f\|_{0,2, \mathbf{R}^{2}}=\|\varphi\|_{0,2, \mathbf{R}^{2}}=1\right\} \\
& \leqslant \sup \left\{\left\|\left(T-T_{h}\right) f\right\|_{0,2 \mathbf{R}^{2}} ;\|f\|_{\left.0,2, \mathbf{R}^{2}=1\right\}}\right. \\
& \leqslant C h^{k+1} \text { according to (4.4). }
\end{aligned}
$$

We then have the following results, for $u \in R(E)$ with $\|u\|_{0,2, \Omega}=1$ and $u_{h}=E_{h} u_{h}$ :

$$
\begin{gathered}
\left\|u-u_{h}\right\|_{0,2, \mathbb{R}^{2}}=O\left(h^{k+1}\right), \\
\left|\lambda-\lambda_{h}\right|=O\left(h^{k+1}\right) .
\end{gathered}
$$

We now turn to the proof of the two propositions stated above.

\section{Proof of Proposition 1}

We first give some notations. We decompose $\Omega \cup \Omega_{h}$ into three domains:

$$
\left\{\begin{array}{l}
\boldsymbol{\Theta}=\boldsymbol{\Omega} \cap \boldsymbol{\Omega}_{h}, \\
\Delta_{i}=\Omega \backslash \boldsymbol{\Theta}, \\
\Delta_{e}=\Omega_{h} \backslash \boldsymbol{\Theta}, \\
\Gamma_{i}=\partial \Omega \cap \bar{\Delta}_{i}, \\
\Gamma_{e}=\partial \Omega \cap \bar{\Delta}_{e} .
\end{array}\right.
$$

We let $\vec{n}=\nu=\left(\nu_{1}, \nu_{2}\right)$ be the unit normal vector, exterior to $\partial \Omega$, and set

$$
\left\{\begin{array}{c}
\partial_{\nu}=\frac{\partial}{\partial \nu}, \\
\partial_{\nu_{L}}=\frac{\partial}{\partial \nu_{L}}=\sum_{i, j=1}^{2} \nu_{i} a_{i j} \frac{\partial}{\partial x_{j}}, \\
\partial_{\nu_{L^{*}}}=\frac{\partial}{\partial \nu_{L^{*}}}=\sum_{i, j=1}^{2} \nu_{j} a_{i j} \frac{\partial}{\partial x_{i}}, \\
A(\sigma)=\sum_{i, j=1}^{2} a_{i j}(x(\sigma)) \nu_{i} \nu_{j} .
\end{array}\right.
$$


5.1. Proof of Proposition 1. We divide the proof of Proposition 1 into two lemmas.

Lemma 5.1. We have the following estimates:

$$
\begin{aligned}
\lambda-\lambda_{h} & =-\lambda^{2} \ell^{*}\left(\left(T-T_{h}\right) u\right)+O\left(h^{2 k+2}\right), \\
\ell^{*}\left(\left(T-T_{h}\right) u\right)= & a_{\Delta_{i}}\left(T u, T^{*} u^{*}\right)+a_{\Delta_{e}}\left(T_{h} u, T_{h}^{*} u^{*}\right) \\
& +a_{\Theta}\left(\left(T-T_{h}\right) u,\left(T^{*}-T_{h}^{*}\right) u^{*}\right) \\
& +\int_{\Gamma_{e}} A(\sigma)\left[\partial_{\nu}(T u) T_{h}^{*} u^{*}+\partial_{\nu}\left(T^{*} u^{*}\right) T_{h} u\right] d \sigma .
\end{aligned}
$$

Remark. The first estimate is a consequence of Theorem 3 in [10] together with (4.4).

We introduce

$$
g(\sigma)=A(\sigma) \partial_{\nu} u \partial_{\nu} u^{*}(x(\sigma)) .
$$

Lemma 5.2. We have the following equalities:

$$
\begin{gathered}
a_{\Delta_{i}}\left(u, u^{*}\right)=-\int_{\Gamma_{i}} g(\sigma) d_{h}(\sigma) d \sigma+O\left(h^{2 k+2}\right), \\
a_{\Delta_{e}}\left(T_{h} u, T_{h}^{*} u^{*}\right)=\frac{1}{\lambda^{2}} \int_{\Gamma_{e}} g(\sigma) d_{h}(\sigma) d \sigma+O\left(h^{2 k}\right), \\
\int_{\Gamma_{e}} A(\sigma) \partial_{\nu}\left(u^{*}\right) T_{h} u d \sigma=-\frac{1}{\lambda} \int_{\Gamma_{e}} g(\sigma) d_{h}(\sigma) d \sigma+O\left(h^{2 k+1}\right), \\
\int_{\Gamma_{e}} A(\sigma) \partial_{\nu}(u) T_{h}^{*} u^{*} d \sigma=-\frac{1}{\lambda} \int_{\Gamma_{e}} g(\sigma) d_{h}(\sigma) d \sigma+O\left(h^{2 k+1}\right) .
\end{gathered}
$$

Suppose for the moment that these lemmas hold. We show that they imply Proposition 1. According to the first lemma, we have:

$$
\begin{aligned}
\lambda-\lambda_{h}= & -\lambda^{2} a_{\Delta_{i}}\left(T u, T^{*} u^{*}\right)-\lambda^{2} a_{\Delta_{e}}\left(T_{h} u, T_{h}^{*} u^{*}\right) \\
& -\lambda^{2} a_{\Theta}\left(\left(T-T_{h}\right) u,\left(T^{*}-T_{h}^{*}\right) u^{*}\right) \\
& -\lambda^{2} \int_{\Gamma_{e}} A(\sigma)\left[\partial_{\nu}(T u) T_{h}^{*} u^{*}+\partial_{\nu}\left(T^{*} u^{*}\right) T_{h} u\right] d \sigma .
\end{aligned}
$$

Using $\lambda T u=u, \lambda T^{*} u^{*}=u^{*}$ and the bilinearity of $a$, we get

$$
\begin{aligned}
\lambda-\lambda_{h}= & -a_{\Delta_{i}}\left(u, u^{*}\right)-\lambda^{2} a_{\Delta_{e}}\left(T_{h} u, T_{h}^{*} u^{*}\right)-\lambda^{2} a_{\Theta}\left(\left(T-T_{h}\right) u,\left(T^{*}-T_{h}^{*}\right) u^{*}\right) \\
& -\lambda \int_{\Gamma_{e}} A(\sigma)\left[\partial_{\nu}(u) T_{h}^{*} u^{*}+\partial_{\nu}\left(u^{*}\right) T_{h} u\right] d \sigma .
\end{aligned}
$$

We then use Lemma 5.2 and obtain

$$
\begin{aligned}
\lambda-\lambda_{h}= & \int_{\Gamma_{i}} g(\sigma) d_{h}(\sigma) d \sigma-\int_{\Gamma_{e}} g(\sigma) d_{h}(\sigma) d \sigma+O\left(h^{2 k}\right) \\
& -\lambda^{2} a_{\Theta}\left(\left(T-T_{h}\right) u,\left(T^{*}-T_{h}^{*}\right) u^{*}\right)+2 \int_{\Gamma_{e}} g(\sigma) d_{h}(\sigma) d \sigma \\
= & \int_{\partial \Omega} g(\sigma) d_{h}(\sigma) d \sigma+O\left(h^{2 k}\right)-\lambda^{2} a_{\Theta}\left(\left(T-T_{h}\right) u,\left(T^{*}-T_{h}^{*}\right) u^{*}\right) \\
= & \int_{\partial \Omega} g(\sigma) d_{h}(\sigma) d \sigma+O\left(h^{2 k}\right) .
\end{aligned}
$$


To obtain the last equality, we have used the continuity of $a_{\theta}$ and the following inequality:

$$
\left\|\left(T-T_{h}\right) u\right\|_{1,2, \Theta}+\left\|\left(T^{*}-T_{h}^{*}\right) u^{*}\right\|_{1,2, \Theta} \leqslant C h^{k},
$$

which is Proposition 1. Now we prove the two lemmas stated above.

5.2. Proof of Lemma 5.1. To show the truth of (1) in Lemma 5.1, we remark that, for all $w \in \operatorname{Im}(I-\lambda T)$, we have

$$
\ell^{*}(w)=0 .
$$

This gives us

$$
\begin{aligned}
0 & =\ell^{*}\left(u_{h}-\lambda_{h} T_{h} u_{h}\right) \\
& =\ell^{*}\left(u_{h}-\lambda T u_{h}\right)+\lambda_{h} \ell^{*}\left(\left(T-T_{h}\right) u_{h}\right)+\left(\lambda-\lambda_{h}\right) \ell^{*}\left(T u_{h}\right) \\
& =\lambda_{h} \ell^{*}\left(\left(T-T_{h}\right) u_{h}\right)+\left(\lambda-\lambda_{h}\right) \ell^{*}\left(T u_{h}\right) \text { according to }(5.4) .
\end{aligned}
$$

Thus,

$$
\left(\lambda-\lambda_{h}\right) \ell^{*}\left(T u_{h}\right)=-\lambda_{h} \ell^{*}\left(\left(T-T_{h}\right) u_{h}\right)
$$

Furthermore,

$$
\begin{aligned}
\ell^{*}\left(T u_{h}\right) & =\ell^{*}(T u)-\ell^{*}\left(T\left(u-u_{h}\right)\right) \\
& =\frac{1}{\lambda}+O\left(h^{k+1}\right) \quad \text { according to }(4.4), \\
\ell^{*}\left(\left(T-T_{h}\right) u_{h}\right) & =\ell^{*}\left(\left(T-T_{h}\right) u\right)-\ell^{*}\left(\left(T-T_{h}\right)\left(u-u_{h}\right)\right) .
\end{aligned}
$$

We recall that the last term satisfies

$$
\begin{aligned}
\ell^{*}\left(\left(T-T_{h}\right)\left(u-u_{h}\right)\right) & =\int_{\Omega}\left(T^{*}-T_{h}^{*}\right) u^{*}\left(u-u_{h}\right) d x \\
& =O\left(h^{2 k+2}\right) \text { according to (4.4) and (4.6). }
\end{aligned}
$$

Equalities (5.5) and (5.6) imply

$$
\lambda-\lambda_{h}=-\lambda \lambda_{h} \ell^{*}\left(\left(T-T_{h}\right) u\right)+O\left(h^{2 k+2}+\left|\lambda-\lambda_{h}\right| h^{k+1}\right),
$$

and we obtain the desired result thanks to (4.4) and (4.7).

The second relation in Lemma 5.1 is a decomposition of the integral $\ell^{*}\left(\left(T-T_{h}\right) u\right)$ over the domains defined in (5.1). From Green's formula,

$$
\begin{gathered}
a_{\Omega}(v, w)-\int_{\Omega} v L^{*} w d x=\int_{\partial \Omega} \partial_{\nu_{L^{*}}} w v d \sigma, \\
a_{\Omega}(w, v)-\int_{\Omega} L v w d x=\int_{\partial \Omega} \partial_{\nu_{L}} v w d \sigma .
\end{gathered}
$$

Choosing $v=T_{h} u$ and $w=T^{*} u^{*}$ in the first one, and $v=T u$ and $w=T_{h}^{*} u^{*}$ in the second one, we have

$$
\begin{aligned}
\ell^{*}\left(T_{h} u\right) & =a_{\Omega}\left(T_{h} u, T^{*} u^{*}\right)-\int_{\partial \Omega} \partial_{\nu_{L^{*}}}\left(T^{*} u^{*}\right) T_{h} u d \sigma, \\
a_{\Omega}\left(T u, T_{h}^{*} u^{*}\right) & =\int_{\partial \Omega} \partial_{\nu_{L}}(T u) T_{h}^{*} u^{*} d \sigma+\int_{\Omega} u T_{h}^{*} u^{*} d x \\
& =\int_{\partial \Omega} \partial_{\nu_{L}}(T u) T_{h}^{*} u^{*} d \sigma+a_{\Omega_{h}}\left(T_{h} u, T_{h}^{*} u^{*}\right)
\end{aligned}
$$

by definition of $T_{h}$. 
We know that $T_{h} u=0$ on $\Gamma_{i}$; the first equality in (5.8) and the definition of $T$ lead to

$$
\begin{aligned}
\ell^{*}\left(\left(T-T_{h}\right) u\right)= & a_{\Omega}\left(T u, T^{*} u^{*}\right)-\ell^{*}\left(T_{h} u\right) \\
= & a_{\Omega}\left(\left(T-T_{h}\right) u, T^{*} u^{*}\right)+\int_{\Gamma_{e}} \partial_{\nu_{L^{*}}}\left(T^{*} u^{*}\right) T_{h} u d \sigma \\
= & a_{\Omega}\left(\left(T-T_{h}\right) u,\left(T^{*}-T_{h}^{*}\right) u^{*}\right)+\int_{\Gamma_{e}} \partial_{\nu_{L^{*}}}\left(T^{*} u^{*}\right) T_{h} u d \sigma \\
& \quad+a_{\Omega}\left(T u, T_{h}^{*} u^{*}\right)-a_{\Omega}\left(T_{h} u, T_{h}^{*} u^{*}\right) \\
= & a_{\Theta}\left(\left(T-T_{h}\right) u,\left(T^{*}-T_{h}^{*}\right) u^{*}\right)+a_{\Delta_{i}}\left(T u, T^{*} u^{*}\right) \\
& \quad+\int_{\Gamma_{e}}\left[\partial_{\nu_{L^{*}}}\left(T^{*} u^{*}\right) T_{h} u+\partial_{\nu_{L}}(T u) T_{h}^{*} u^{*}\right] d \sigma \\
& +a_{\Delta_{e}}\left(T_{h} u, T_{h}^{*} u^{*}\right) \text { according to (4.8). }
\end{aligned}
$$

The proof of the second relation is complete after we note that

$$
\begin{aligned}
\partial_{\nu_{L^{*}}}\left(T^{*} u^{*}\right) & =A(\sigma) \partial_{\nu} T^{*} u^{*}, \\
\partial_{\nu_{L}}(T u) & =A(\sigma) \partial_{\nu} T u,
\end{aligned}
$$

since $T u=T^{*} u^{*}=0$ on $\partial \Omega$.

5.3. Proof of Lemma 5.2. Proof of (1): we describe $\partial \Omega_{h}$ with the notation defined in (3.8). Every point $y$ of $\Delta_{i}$ can be written in a unique way as follows:

$$
y=x(\sigma)+\xi \vec{n}(\sigma) \text { with } \xi \in\left(d_{h}(\sigma), 0\right)
$$

Taylor's formula at the point $x(\sigma)$ gives

$$
\begin{aligned}
\frac{\partial u}{\partial x_{i}}(y) \frac{\partial u^{*}}{\partial x_{j}}(y) & =\frac{\partial u}{\partial x_{i}}(x(\sigma)) \frac{\partial u^{*}}{\partial x_{j}}(x(\sigma))+O\left(h^{k+1}\right) \text { according to Corollary } 3.1 \\
& =\nu_{i} \nu_{j} \frac{\partial u}{\partial \nu}(x(\sigma)) \frac{\partial u^{*}}{\partial \nu}(x(\sigma))+O\left(h^{k+1}\right) \text { because of } u=0 \text { on } \Gamma_{i} .
\end{aligned}
$$

We note that

$$
d x_{1} d x_{2}=\left(1-\frac{\xi}{R(\sigma)}\right) d \sigma d \xi=\left(1+O\left(h^{k+1}\right)\right) d \sigma d \xi,
$$

where $R(\sigma)$ is the radius of curvature of $\partial \Omega$ at the point $x(\sigma)$. The second equality is a consequence of Corollary 3.1. This gives us

$$
\begin{aligned}
a_{\Delta_{i}}\left(u, u^{*}\right) & =\int_{\Gamma_{i}} \sum_{i, j=1}^{2} \int_{d_{h}(\sigma)}^{0}\left(a_{i j} \nu_{i} \nu_{j} \frac{\partial u}{\partial \nu} \frac{\partial u^{*}}{\partial \nu}+O\left(h^{k+1}\right)\right) d \xi d \sigma \\
& =-\int_{\Gamma_{i}} A(\sigma) \frac{\partial u}{\partial \nu} \frac{\partial u^{*}}{\partial \nu}(x(\sigma)) d_{h}(\sigma) d \sigma+O\left(h^{2 k+2}\right),
\end{aligned}
$$

which ends the proof of the first relation.

Proof of (2): for the sake of simplicity, we let

$$
\left\{\begin{array} { l } 
{ v _ { h } = T _ { h } u , } \\
{ v _ { h } ^ { * } = T _ { h } ^ { * } u ^ { * } }
\end{array} \text { and } \left\{\begin{array}{ll}
v=T u & \text { on } \Omega, \\
v^{*}=T^{*} u^{*} & \text { on } \Omega .
\end{array}\right.\right.
$$


The boundary of $\Omega$ being regular, we can extend $v$ and $v^{*}$ to $\mathbb{R}^{2} \backslash \Omega$ as $C^{k+1}$-functions. The bilinearity of $a_{\Delta_{e}}$ gives us

$$
\begin{aligned}
a_{\Delta_{e}}\left(v_{h}, v_{h}^{*}\right)= & a_{\Delta_{e}}\left(v, v^{*}\right)+a_{\Delta_{e}}\left(v_{h}-v, v^{*}\right)+a_{\Delta_{e}}\left(v, v_{h}^{*}-v^{*}\right) \\
& +a_{\Delta_{e}}\left(v_{h}-v, v_{h}^{*}-v^{*}\right) .
\end{aligned}
$$

Analogously to the previous argument, we have

$$
a_{\Delta_{e}}\left(v, v^{*}\right)=\int_{\Gamma_{e}} \tilde{g}(\sigma) d_{h}(\sigma) d \sigma+O\left(h^{2 k+2}\right)
$$

with $\widetilde{g}(\sigma)=A(\sigma) \partial_{\nu} v \partial_{\nu} v^{*}(x(\sigma))$, hence $\lambda^{2} \widetilde{g}(\sigma)=g(\sigma)$, and we obtain the first term given in the equality we are trying to prove. We now show that the remaining terms in the equality (5.10) are bounded by $h^{2 k}$. We first use the continuity of $a_{\Delta_{e}}$ :

$$
\begin{gathered}
\left|a_{\Delta_{e}}\left(v-v_{h}, v^{*}\right)\right| \leqslant C\left\|v-v_{h}\right\|_{1,2, \Delta_{e}}\left\|v^{*}\right\|_{1,2, \Delta_{e}}, \\
\left|a_{\Delta_{e}}\left(v, v^{*}-v_{h}^{*}\right)\right| \leqslant C\left\|v^{*}-v_{h}^{*}\right\|_{1,2, \Delta_{e}}\|v\|_{1,2, \Delta_{e},}, \\
\left|a_{\Delta_{e}}\left(v-v_{h}, v^{*}-v_{h}^{*}\right)\right| \leqslant C\left\|v-v_{h}\right\|_{1,2, \Delta_{e}}\left\|v^{*}-v_{h}^{*}\right\|_{1,2, \Delta_{e}} .
\end{gathered}
$$

Let $r_{h} v$ be the Lagrangian interpolation polynomial of degree $k$ of $v$. According to Ciarlet and Raviart [8], we have

$$
\left\|v-r_{h} v\right\|_{1,2, \Omega_{h}} \leqslant C h^{k} .
$$

According to (4.2), we then obtain

$$
\left\|v_{h}-r_{h} v\right\|_{1,2, \Theta} \leqslant C h^{k} .
$$

Using the fact that $v_{h}-r_{h} v$ belongs to $V_{h}$ and Lemma 3.3, we furthermore have

$$
\begin{aligned}
\left\|v_{h}-r_{h} v\right\|_{1,2, \Delta_{e}} & \leqslant C h^{\frac{k}{2}}\left\|v_{h}-r_{h} v\right\|_{1,2, \Theta} \\
& \leqslant C h^{\frac{3 k}{2}} \quad \text { according to (5.14). }
\end{aligned}
$$

We also have, by Ciarlet and Raviart [8],

$$
\left\|v-r_{h} v\right\|_{1, \infty, \Omega \cup \Omega_{h}} \leqslant C h^{k} .
$$

It is then clear that we obtain

$$
\begin{aligned}
\left\|v-r_{h} v\right\|_{1,2, \Delta_{e}} & \leqslant\left(\operatorname{area}\left(\Delta_{e}\right)\right)^{\frac{1}{2}}\left\|v-r_{h} v\right\|_{1, \infty, \Delta_{e}} \\
& \leqslant C h^{\frac{3}{2} k+\frac{1}{2} .}
\end{aligned}
$$

Hence, we obtain

$$
\left\|v-v_{h}\right\|_{1,2, \Delta_{e}} \leqslant C h^{\frac{3}{2} k} .
$$

The same kind of estimate holds for $v^{*}-v_{h}^{*}$. We furthermore have the following inequality:

$$
\left\|v^{*}\right\|_{1,2, \Delta_{e}} \leqslant C\left(\operatorname{area}\left(\Delta_{e}\right)\right)^{\frac{1}{2}} \leqslant C h^{\frac{k+1}{2}},
$$

which is also true for $\|v\|_{1,2, \Delta_{e}}$. 
Putting these two last results in (5.12), we have

$$
\begin{aligned}
\left|a_{\Delta_{e}}\left(v-v_{h}, v^{*}\right)\right| & \leqslant C h^{2 k+\frac{1}{2}}, \\
\left|a_{\Delta_{e}}\left(v, v^{*}-v_{h}^{*}\right)\right| & \leqslant C h^{2 k+\frac{1}{2}}, \\
\left|a_{\Delta_{e}}\left(v-v_{h}, v^{*}-v_{h}^{*}\right)\right| & \leqslant C h^{3 k} .
\end{aligned}
$$

Using these inequalities in the equality (5.10), we obtain the second relation of Lemma 5.2, thanks to (5.11).

Proof of (3): the proof of (4) being similar, it will be omitted. We use the notations (5.9). The function $v_{h}$ vanishes on $\partial \Omega_{h}$, hence, according to (3.8), we can write

$$
\begin{aligned}
v_{h}(x(\sigma))= & -d_{h}(\sigma) \vec{n}(\sigma) \nabla v_{h}(y) \quad \text { with } y \in\left(x(\sigma), x_{h}(\sigma)\right) \\
= & -d_{h}(\sigma) \vec{n}(\sigma) \nabla v_{h}(x(\sigma))+d_{h}(\sigma) \vec{n}(\sigma)\left(\nabla v_{h}(x(\sigma))-\nabla v_{h}(y)\right) \\
= & -d_{h}(\sigma) \partial_{\nu} v_{h}(x(\sigma))+O\left(h^{k+1}\left|\vec{n}(\sigma)\left(\nabla v_{h}(x(\sigma))-\nabla v_{h}(y)\right)\right|\right) \\
& \quad \text { according to the estimate on } d_{h} \text { obtained in Corollary 3.1. }
\end{aligned}
$$

We furthermore have

$$
\begin{aligned}
\mid \vec{n}(\sigma) & \left(\nabla v_{h}(x(\sigma))-\nabla v_{h}(y)\right) \mid \\
\leqslant & \left|\vec{n}(\sigma)\left(\nabla v_{h}(x(\sigma))-\nabla v(x(\sigma))\right)\right| \\
& +|\vec{n}(\sigma)(\nabla v(x(\sigma))-\nabla v(y))| \\
& +\left|\vec{n}(\sigma)\left(\nabla v_{h}(y)-\nabla v(y)\right)\right| \\
\leqslant & C\left(\left\|v-v_{h}\right\|_{1,2, \Delta_{e}}+|x(\sigma)-y|\right) \\
\leqslant & C h^{k+1} \text { according to }(5.17) \text { and Corollary 3.1, }
\end{aligned}
$$

which gives

$$
v_{h}(x(\sigma))=-d_{h}(\sigma) \partial_{\nu} v_{h}(x(\sigma))+O\left(h^{2 k+2}\right) .
$$

We finally obtain

$$
\int_{\Gamma_{e}} A(\sigma) \partial_{\nu}\left(u^{*}\right) T_{h} u d \sigma=-\int_{\Gamma_{e}} A(\sigma) \partial_{\nu}\left(u^{*}\right) d_{h}(\sigma) \partial_{\nu} v_{h}(x(\sigma)) d \sigma+O\left(h^{2 k+2}\right) .
$$

Since $\left\|v-v_{h}\right\|_{1,2, \Theta}=O\left(h^{k+1}\right)$, we have

$$
\partial_{\nu} v_{h}(x(\sigma))=\partial_{\nu} v(x(\sigma))+O\left(h^{k+1}\right) .
$$

Furthermore,

$$
\left\{\begin{array}{l}
v=T u=\frac{1}{\lambda} u \\
d_{h}(\sigma)=O\left(h^{k+1}\right)
\end{array}\right.
$$

Therefore,

$$
d_{h}(\sigma) \partial_{\nu} v_{h}(x(\sigma))=\frac{1}{\lambda} d_{h}(\sigma) \partial_{\nu} u(x(\sigma))+O\left(h^{2 k+2}\right)
$$

This, and (5.18), yield the proof of (3) and the lemma is completely proved. 


\section{Proof of Proposition 2}

We use the Gauss-Lobatto formula to prove the proposition. Therefore, we introduce $\theta_{0}=0, \theta_{1}, \ldots, \theta_{k}=1$, the $k+1$ Gauss-Lobatto quadrature points of $[0,1]$, and

$$
G_{i}(f)=\sum_{j=0}^{k} l_{j} \lambda_{j} f\left(\sigma_{i}+\theta_{j} l_{i}\right),
$$

where the coefficients $\lambda_{i}$ are uniquely determined by

$$
G_{i}(p)=\int_{0}^{1} p(x) d x \text { for all } p \in P_{2 k-1} \text {. }
$$

We recall that $\lambda_{j}>0$ and $\sum_{j=0}^{k} \lambda_{j}=1$.

According to the Peano theorem (see, for example, [9]), we have

$$
\text { For all } f \in C^{2 k}\left(\left[\sigma_{i}, \sigma_{i+1}\right]\right) \quad\left|E_{i}(f)\right| \leqslant C l_{i}^{2 k+1}|f|_{2 k, \infty, \Gamma},
$$

where

$$
E_{i}(f)=\int_{\sigma_{i}}^{\sigma_{i+1}} f(\sigma) d \sigma-G_{i}(f) .
$$

We now begin the proof of Proposition 2. Denote $\gamma_{i}=\left[\sigma_{i}, \sigma_{i+1}\right]$ and consider a $W^{k-1,1}(\partial \Omega)$-function $\varphi$. Then we have, for all $\sigma \in \gamma_{i}$,

$$
\varphi(\sigma)=p_{i}(\sigma)+\int_{\sigma_{i}}^{\sigma} \varphi^{(k-1)}(s) \frac{(\sigma-s)^{k-2}}{(k-2) !} d s,
$$

where $p_{i}$ is Taylor's polynomial of degree $k-2$ of $\varphi$ at the point $\sigma_{i}$. This equality implies that

$$
\left\|\varphi-p_{i}\right\|_{0, \infty, \gamma_{i}} \leqslant C h^{k-2}|\varphi|_{k-1,1, \gamma_{i}} .
$$

Furthermore, we can write:

$$
\int_{\gamma_{i}} \varphi(\sigma) d_{h}(\sigma) d \sigma=\int_{y_{i}}\left(\varphi-p_{i}\right)(\sigma) d_{h}(\sigma) d \sigma+E_{i}\left(p_{i} d_{h}\right)+l_{i} \sum_{j=0}^{k} \lambda_{j}\left(p_{i} d_{h}\right)\left(\sigma_{i, j}\right) .
$$

According to (6.4), (6.6), and Lemma 3.1, we deduce

$$
\begin{aligned}
& \left|\int_{\gamma_{i}} \varphi(\sigma) d_{h}(\sigma) d \sigma\right| \\
& \quad \leqslant C\left(h^{2 k}|\varphi|_{k-1,1, \gamma_{i}}+l_{i} h^{2 k}\left|p_{i} d_{h}\right|_{2 k, \infty, \gamma_{i}}+l_{i} \max _{j=0, \cdots, k}\left|\left(p_{i} d_{h}\right)\left(\sigma_{i, j}\right)\right|\right) \\
& \quad \leqslant C\left(h^{2 k}|\varphi|_{k-1,1, \gamma_{i}}+l_{i}\|\varphi\|_{k-2, \infty, \gamma_{i}}\left(h^{2 k}\left|d_{h}\right|_{2 k, \infty, \gamma_{i}}+\max _{j=0, \cdots, k}\left|d_{h}\left(\sigma_{i, j}\right)\right|\right)\right),
\end{aligned}
$$

where we have used the inequalities:

$$
\begin{gathered}
\max _{j=0, \cdots, k}\left|p_{i}\left(\sigma_{i, j}\right)\right| \leqslant\left\|p_{i}\right\|_{0, \infty, \gamma_{i}} \leqslant C\|\varphi\|_{k-2, \infty, \gamma_{i}}, \\
\left|p_{i} d_{h}\right|_{2 k, \infty, \gamma_{i}} \leqslant C\|\varphi\|_{k-2, \infty, \gamma_{i}}\left\|d_{h}\right\|_{2 k, \infty, \gamma_{i}} .
\end{gathered}
$$


Carrying out the summation over all intervals $\gamma_{i}$, we obtain

$$
\begin{aligned}
& \left|\int_{\partial \Omega} \varphi(\sigma) d_{h}(\sigma) d \sigma\right| \\
& \leqslant C\left(h^{2 k}|\varphi|_{k-1,1, \partial \Omega}\right. \\
& \left.\quad+L\|\varphi\|_{k-2, \infty, \partial \Omega}\left(\max _{j, i}\left|d_{h}\left(\sigma_{i, j}\right)\right|+h^{2 k} \max _{i}\left\|d_{h}\right\|_{2 k, \infty, \gamma_{i}}\right)\right) .
\end{aligned}
$$

To complete the proof of Proposition 2, we need the following lemma.

Lemma 6.1. There is a nonnegative constant $C$ such that, for all $i$, we have

$$
\left\{\begin{aligned}
\left\|d_{h}\right\|_{2 k, \infty, \gamma_{i}} & \leqslant C, \\
\left|d_{h}\left(\sigma_{i, j}\right)\right| & \leqslant C\left(\left|\left(x-x_{h}\right)\left(\sigma_{i, j}\right)\right|+h^{2 k+1}\right) .
\end{aligned}\right.
$$

Proof of Lemma 6.1. We construct two parametric representations of $\partial \Omega_{h}$ :

$$
\begin{gathered}
s \in\left[\sigma_{i}, \sigma_{i+1}\right] \rightarrow x_{h}(s)=F_{K}\left(\frac{s-\sigma_{i}}{l_{i}}, 0\right), \\
\sigma \in\left[\sigma_{i}, \sigma_{i+1}\right] \rightarrow \widetilde{x}_{h}(\sigma)=x(\sigma)+d_{h}(\sigma) \vec{n}(\sigma) .
\end{gathered}
$$

This defines a homeomorphism

$$
f: \sigma \in[0, L] \rightarrow s=f(\sigma) \in[0, L]
$$

with

$$
x_{h}(s)=\tilde{x}_{h}(\sigma)=x(\sigma)+d_{h}(\sigma) \vec{n}(\sigma),
$$

which gives us

$$
d_{h}(\sigma)=\left(\left(x_{h}(s)-x(\sigma)\right) \cdot \vec{n}(\sigma)\right) .
$$

We already observed that $x_{h}$ and all its derivatives are bounded independently of $h$ and $i$; then we obtain the first inequality, provided $f$ is $C^{\infty}$ on $[0, L]$ and has all its derivatives bounded independently of $h$ and $i$. We first prove with a Taylor expansion that there is a constant $C$ independent of $i$ and $h$ such that

$$
|\sigma-s| \leqslant C h^{k+1}
$$

According to (6.7) we have

$$
\begin{aligned}
x_{h}(s)-x(s) & =x(\sigma)-x(s)+d_{h}(\sigma) \vec{n}(\sigma), \\
& =(s-\sigma) \vec{t}(\sigma)+\left(\frac{(s-\sigma)^{2}}{2 R(\sigma)}+d_{h}(\sigma)\right) \vec{n}(\sigma)+O\left(|s-\sigma|^{3}\right),
\end{aligned}
$$

where $R(\sigma)$ is the radius of curvature of $\partial \Omega$ at the point $x(\sigma)$.

Lemma 3.1 and Corollary 3.1 say that we have $\left\|x-x_{h}\right\|_{0, \infty, \partial \Omega} \leqslant C h^{k+1}$ and $d_{h}(\sigma) \leqslant C h^{k+1}$, thus (6.10) leads to (6.9).

We now study the regularity of $f$. According to (6.7) we have

$$
f(\sigma)=l_{i} F_{K}^{-1}\left(x(\sigma)+d_{h}(\sigma) \vec{n}(\sigma)\right)+\sigma_{i} .
$$


We assume the triangulation to be $k$-regular; this implies that $F_{K}$ is a $C^{k}$ diffeomorphism; furthermore, it belongs to $\left(P_{k}\right)^{2}$. It is then clearly a $C^{\infty}$ diffeomorphism. Since $d_{h}$ is regular and using (6.11), we obtain that $f$ is regular.

In order to prove that all derivatives of $f$ are bounded independently of $h$ on $[0, L]$, we multiply $(5.7)$ by $x^{\prime}(\sigma)=\vec{t}(\sigma)$, and we have

$$
\phi(\sigma) \stackrel{\text { def }}{=}\left(x(\sigma) \cdot x^{\prime}(\sigma)\right)=\left(x_{h}(s) \cdot x^{\prime}(\sigma)\right),
$$

where $\phi$ is a $C^{\infty}$-function on $[0, L]$ which does not depend on $h$. Carrying out the differentiation with respect to $\sigma$, we obtain

$$
\phi^{\prime}(\sigma)=f^{\prime}(\sigma)\left(x_{h}^{\prime}(s) \cdot x^{\prime}(\sigma)\right)+\left(x_{h}(s) \cdot x^{\prime \prime}(\sigma)\right) .
$$

According to (6.9), we can write

$$
\begin{aligned}
\left(x_{h}^{\prime}(s) \cdot x^{\prime}(\sigma)\right) & =\left(x^{\prime}(\sigma) \cdot x^{\prime}(\sigma)\right)+\left(x^{\prime}(\sigma) \cdot x^{\prime}(s)-x^{\prime}(\sigma)\right)+\left(x^{\prime}(\sigma) \cdot x_{h}^{\prime}(s)-x^{\prime}(s)\right) \\
& =1+O\left(h^{k}\right),
\end{aligned}
$$

because of $\left\|x^{\prime}-x_{h}^{\prime}\right\|_{0, \infty, \partial \Omega}=O\left(h^{k}\right)$ as a consequence of Lemma 3.1. We deduce from these calculations that, if $h$ is small enough,

$$
\left(x_{h}^{\prime}(s) \cdot x^{\prime}(\sigma)\right) \neq 0
$$

thus $f^{\prime}$ is independent of $h$ since $x_{h}$ and all its derivatives also are. We then obtain that all derivatives of $f$ are bounded independently of $h$ thanks to the previous remark and with the help of an induction by carrying out the differentiation of the equation (6.13) with respect to $\sigma$. Thus, all the derivatives of $f$ are bounded independently of $h$ on $[0, L]$ and inequality (6.8) proves the first part of the lemma. We now proceed to the second part.

Define

$$
s_{i, j}=f\left(\sigma_{i, j}\right),
$$

and write (5.8) at the points $s_{i, j}$ and $\sigma_{i, j}$ :

$$
\begin{aligned}
d_{h}\left(\sigma_{i, j}\right)= & \left(\left(x_{h}\left(s_{i, j}\right)-x\left(\sigma_{i, j}\right)\right) \cdot \vec{n}\left(\sigma_{i, j}\right)\right) \\
= & \left(\left(x_{h}\left(\sigma_{i, j}\right)-x\left(\sigma_{i, j}\right)\right) \cdot \vec{n}\left(\sigma_{i, j}\right)\right)+\left(\left(x_{h}\left(s_{i, j}\right)-x_{h}\left(\sigma_{i, j}\right)\right) \cdot \vec{n}\left(\sigma_{i, j}\right)\right) \\
& +\left(\left(x\left(\sigma_{i, j}\right)-x\left(s_{i, j}\right)\right) \cdot \vec{n}\left(\sigma_{i, j}\right)\right) .
\end{aligned}
$$

We know that

$$
\begin{aligned}
x_{h}\left(s_{i, j}\right)-x_{h}\left(\sigma_{i, j}\right)= & \left(s_{i, j}-\sigma_{i, j}\right) x_{h}^{\prime}\left(\sigma_{i, j}\right)+O\left(h^{2 k+2}\right) \\
& \text { according to the estimate of } s-\sigma \text { stated in }(6.9) \\
= & \left(s_{i, j}-\sigma_{i, j}\right) x^{\prime}\left(\sigma_{i, j}\right)+O\left(h^{2 k+2}\right) \\
& +\left(s_{i, j}-\sigma_{i, j}\right)\left(x_{h}^{\prime}\left(\sigma_{i, j}\right)-x^{\prime}\left(\sigma_{i, j}\right)\right) \\
= & \left(s_{i, j}-\sigma_{i, j}\right) x^{\prime}\left(\sigma_{i, j}\right)+O\left(h^{2 k+1}\right) \text { since }\left\|x-x_{h}\right\|=O\left(h^{k}\right) .
\end{aligned}
$$

Substituting the last equality into (6.15), we obtain

$$
d_{h}\left(\sigma_{i, j}\right)=\left(\left(x_{h}\left(\sigma_{i, j}\right)-x\left(\sigma_{i, j}\right)\right) \cdot \vec{n}\left(\sigma_{i, j}\right)\right)+O\left(h^{2 k+1}\right),
$$

because of $x^{\prime}\left(\sigma_{i, j}\right)=\vec{t}\left(\sigma_{i, j}\right)$. 
Remark. According to (6.15), we could change $\left(H_{2}\right)$ and $(H)$ to $\left(H_{3}\right)$ :

$$
\left(H_{3}\right) \quad\left\{\begin{array}{l}
\left|\left(\left(x_{h}\left(\sigma_{i, j}\right)-x\left(\sigma_{i, j}\right)\right) \cdot \vec{n}\left(\sigma_{i, j}\right)\right)\right| \leqslant C h^{2 k}, \\
\left|x_{h}\left(\sigma_{i, j}\right)-x\left(\sigma_{i, j}\right)\right| \leqslant C l_{i}^{k+1} .
\end{array}\right.
$$

\section{EXAMPLES}

We use again the notations of $\S 3$. We consider a triangle $K$ of the triangulation $\mathscr{K}_{h}$ with a curved edge $\Gamma_{h}$ in $\partial \Omega_{h}$ and denote by $A$ and $B$ the vertices of $\Gamma_{h}$. We call $\Gamma$ the part of $\partial \Omega$ lying between these two points. Let $O$ be the midpoint of $A$ and $B$.

For $k=2$, we give two different constructions of the arc $\Gamma_{h}$; for $k=3$, we only give a sketch, since it is the same idea.

7.1. The case $k=2$. The Gauss-Lobatto quadrature points of the segment $[0,1]$, for $k=2$, are $0,1 / 2,1$. We assume that $A$ and $B$ have $-l / 2$ and $l / 2$ as arclength. Let

$$
C^{\prime}=x(0) \text {. }
$$

If we define $\Gamma_{h}$ by the three points $A, B$, and $C^{\prime}$, then the hypothesis $(H)$ clearly holds, and the triangle $K$ is $k$-regular, but $C^{\prime}$ is difficult to calculate if $\Gamma$ is not parametrized by its arclength.

We can also consider the point $C$ intersection of $\Gamma$ and the median of $[A, B]$. Let us show why this point is convenient. We must have

$$
\overrightarrow{C C^{\prime}}=O\left(h^{4}\right)
$$

Let

$$
\left\{\begin{aligned}
\vec{t}(\sigma) & =\frac{d x(\sigma)}{d \sigma}, \\
C & =x\left(\sigma_{1}\right) .
\end{aligned}\right.
$$

Lemma 7.1. With the previous notations, we have

(1) $\sigma_{1}=O\left(h^{4}\right)$,

(2) $\overrightarrow{C C^{\prime}}=O\left(h^{4}\right)$.

Proof. The point $\sigma_{1}$ is defined by $\overrightarrow{O C} \cdot \overrightarrow{A B}=0$.

We write the expansion of the function $x$ at the point 0 for $\sigma=\sigma_{1},-l / 2$, or $l / 2$,

$$
x(\sigma)=x(0)+\sigma x^{\prime}(0)+\frac{\sigma}{2}^{2} x^{\prime \prime}(0)+\frac{\sigma}{6}^{3} x^{\prime \prime \prime}(0)+O\left(l^{4}\right),
$$

hence

$$
\begin{aligned}
\overrightarrow{O C} & =x\left(\sigma_{1}\right)-\frac{1}{2}\left(x\left(-\frac{l}{2}\right)+x\left(\frac{l}{2}\right)\right) \\
& =\sigma_{1} x^{\prime}(0)+\frac{1}{2}\left(\sigma_{1}^{2}-l^{2}\right) x^{\prime \prime}(0)+\frac{1}{6} \sigma_{1}^{3} x^{\prime \prime \prime}(0)+O\left(l^{4}\right), \\
\overrightarrow{A B} & =x\left(\frac{l}{2}\right)-x\left(-\frac{l}{2}\right)=l\left(x^{\prime}(0)+\frac{1}{36} x^{\prime \prime \prime}(0)+O\left(l^{4}\right)\right) .
\end{aligned}
$$




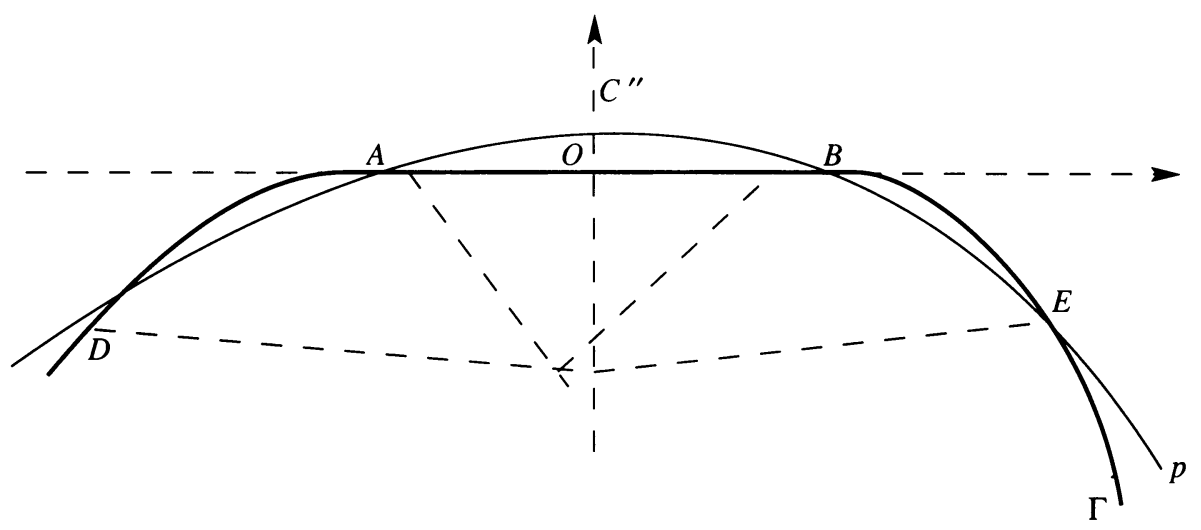

FIGURE 7.1

Thus,

$$
\sigma_{1}+\frac{1}{6} \sigma_{1}^{3}+\frac{1}{36} \sigma_{1} l^{2}\left(x^{\prime}(0), x^{\prime \prime \prime}(0)\right)+O\left(l^{4}\right)=0 .
$$

We deduce that $\sigma_{1}=O\left(l^{4}\right)$. We have already remarked that $l=O(h)$, thus we have shown the first relation of the lemma.

We also have

$$
\overrightarrow{C C^{\prime}}=x\left(\sigma_{1}\right)-x(0)=\sigma_{1} \vec{t}(0)+O\left(h^{8}\right),
$$

which shows the second relation.

The point $C$ satisfies the hypothesis $(H)$; it also satisfies the hypotheses needed for a $k$-regular triangulation ([7]). We remark that any point $C^{\prime \prime}$ with $\vec{C}^{\prime} C^{\prime \prime}=O\left(h^{4}\right)$ is also convenient; we then show another way of constructing the third point required to obtain $\Gamma_{h}$.

Let $D$ and $E$ be the two exterior nodes of the triangulation which are respectively the nearest of $A$ and $B$.

We consider $p$ a polynomial of degree three, passing through $A, B, D$, and $E$, and we denote by $C^{\prime \prime}$ the intersection of $p$ with the median of $[A, B]$; by construction, $C^{\prime \prime}$ satisfies that $\vec{C}^{\prime \prime} C^{\prime \prime}=O\left(h^{4}\right)$ and $C^{\prime \prime}$ is easy to calculate.

We give an algorithm to obtain $C^{\prime \prime}$. We first work with the orthonormal frame of reference defined by Figure 7.1, and we denote by $\left(x_{M}, y_{M}\right)$ the coordinates of a point $M$ in this frame of reference. We have

$$
\left\{\begin{array}{l}
x_{A}=-x_{B}, \\
y_{A}=y_{B}=0, \\
y_{C^{\prime \prime}}=0 .
\end{array}\right.
$$

We define two polynomials $p_{D}$ and $p_{E}$ as follows:

$$
\begin{aligned}
& p_{D}(x)=\frac{\left(x-x_{E}\right)\left(x-x_{A}\right)\left(x-x_{B}\right)}{\left(x_{D}-x_{E}\right)\left(x_{D}-x_{A}\right)\left(x_{D}-x_{B}\right)}, \\
& p_{E}(x)=\frac{\left(x-x_{D}\right)\left(x-x_{A}\right)\left(x-x_{B}\right)}{\left(x_{E}-x_{D}\right)\left(x_{E}-x_{A}\right)\left(x_{E}-x_{B}\right)} .
\end{aligned}
$$


We then define $C^{\prime \prime}$ by

$$
x_{C^{\prime \prime}}=y_{D} p_{D}(0)+y_{E} p_{E}(0) .
$$

We now work in the original frame of reference, assumed to be orthonormal, and denote by $\left(x_{M}^{\prime}, y_{M}^{\prime}\right)$ the coordinates of a point $M$ in this frame of reference; we can then give an algorithm to calculate $C^{\prime \prime}$ :

(1) Change of frame of reference:

$$
\begin{aligned}
& \alpha=\frac{x_{O}^{\prime}-x_{A}^{\prime}}{h / 2}, \quad \beta=\frac{y_{O}^{\prime}-y_{A}^{\prime}}{h / 2}, \\
& f(x, y)=\beta\left(x-x_{o}^{\prime}\right)+\alpha\left(y-y_{o}^{\prime}\right), \\
& g(x, y)=\alpha\left(x-x_{O}^{\prime}\right)-\beta\left(y-y_{O}^{\prime}\right) \text {, } \\
& \left\{\begin{array} { l } 
{ x _ { E } = g ( x _ { E } ^ { \prime } , y _ { E } ^ { \prime } ) , } \\
{ y _ { E } = f ( x _ { E } ^ { \prime } , y _ { E } ^ { \prime } ) }
\end{array} \quad \left\{\begin{array}{l}
x_{D}=g\left(x_{D}^{\prime}, y_{D}^{\prime}\right), \\
y_{D}=f\left(x_{D}^{\prime}, y_{D}^{\prime}\right)
\end{array}\right.\right.
\end{aligned}
$$

(2) Equality (7.6):

$$
\left\{\begin{aligned}
p(x, y, z, t) & =\frac{h^{2}}{4(x-y)}\left[\frac{x z}{x^{2}-h^{2} / 4}-\frac{y t}{y^{2}-h^{2} / 4}\right] \\
c & =p\left(x_{D}, x_{E}, y_{D}, y_{E}\right) .
\end{aligned}\right.
$$

(3) Result:

$$
\left\{\begin{array}{l}
x_{C^{\prime \prime}}^{\prime}=\beta c+x_{O}^{\prime} \\
y_{C^{\prime \prime}}^{\prime}=\alpha c+y_{O}^{\prime} .
\end{array}\right.
$$

Remark. In the case of $k=2$, according to Ciarlet and Raviart [7], the triangulation is $k$-regular if we have $\left\|\overrightarrow{O C^{\prime \prime}}\right\|=O\left(h^{2}\right)$, which is the case; we then construct the other two edges to obtain the other hypotheses of $k$-regularity.

7.2. The case $k=3$. The Gauss-Lobatto quadrature points of the interval $[0,1]$ in the case $k=3$ are $0, \alpha=\frac{1}{2}\left(1-\frac{1}{\sqrt{5}}\right), \beta=\frac{1}{2}\left(1+\frac{1}{\sqrt{5}}\right), 1$. Let

$$
\begin{aligned}
& B=x(\alpha l), \\
& C=x(\beta l) .
\end{aligned}
$$

We then observe that all points $B^{\prime}$ and $C^{\prime}$ satisfying

$$
\begin{aligned}
\left\|\overrightarrow{B B^{\prime}}\right\| & =O\left(h^{6}\right), \\
\left\|\overrightarrow{C C^{\prime}}\right\| & =O\left(h^{6}\right)
\end{aligned}
$$

are convenient to construct $\Gamma_{h}$. We consider a polynomial $p$ of degree five passing through six exterior and nearest nodes of the triangulation and we denote by $B^{\prime}$ (respectively $C^{\prime}$ ) the intersection of $p$ with the orthogonal straight line to $(A, B)$ passing through the point $\alpha A+(1-\alpha) B$ (respectively $\beta A+(1-\beta) B$ ).

These points define a convenient arc $\Gamma_{h}$; we then construct the two other edges of the triangle in order to have a 3-regular triangulation.

\section{ACKNOWLEDGMENT}

This work was suggested by Professor M. Crouzeix and Professor J. Rappaz. The author is greatly indebted to them for many stimulating discussions and 
comments, which have influenced the contents of this paper. She is grateful to the referee for his helpful comments.

\section{BIBLIOGRAPHY}

1. S. Agmon, Lectures on elliptic boundary value problems, Van Nostrand Math. Stud., vol. 2, 1965.

2. I. Babuška and J. E Osborn, Eigenvalue problems, Handbook of Numerical Analysis (P. G. Ciarlet and J. L. Lyons, eds.), vol. 2, North-Holland, Amsterdam, 1991.

3. U. Banerjee, A note on the effect of numerical quadrature in finite element eigenvalue approximation, Numer. Math. 61 (1992), 145-152.

4. U. Banerjee and J. E. Osborn, Estimation in the effect of numerical integration in finite element eigenvalue approximation, Numer. Math. 56 (1990), 735-762.

5. G. Birkhoff, C. De Boor, B.J. Swartz, and B. Wendroff, Rayleigh-Ritz approximation by piecewise cubic polynomials, SIAM J. Numer. Anal. 3 (1966), 188-203.

6. P.G Ciarlet, The finite element method for elliptic problems, North-Holland, Amsterdam, 1978.

7. P. G. Ciarlet and P.A. Raviart, The combined effect of curved boundaries and numerical integration in isoparametric finite element methods, The Mathematical Foundations of the Finite Element Method, with Applications to Partial Differential Equations (A. K. Aziz, ed.), Academic Press, 1973, pp. 409-474.

8. __ Interpolation over curved elements, with applications to finite element methods, Comput. Methods Appl. Mech. Engrg. 1 (1972), 217-249.

9. A. H. Stroud and D. Secrest, Gaussian quadrature formulas, Prentice-Hall, Englewood Cliffs, NJ, 1966.

10. J. E. Osborn, Spectral approximation for compact operators, Math. Comp. 29 (1975), 712725.

11. A. Zenisek, Discrete forms of Friedrich inequalities in the finite element method, RAIRO Numer. Anal. 15 (1981), 265-286.

12. M. Zlámal, Curved elements in the finite element method. I, SIAM J. Numer. Anal. 10 (1973), 229-240.

13. _ Curved elements in the finite element method. II, SIAM J. Numer. Anal. 11 (1974), 347-368.

Universite de Rennes I, Campus de Beaulieu 35042 Rennes Cedex, France

E-mail address: mplebaud@lie.univ-rennes1.fr 